\title{
LAW OF LARGE NUMBERS AND CENTRAL LIMIT THEOREM FOR INDEPENDENT AND NON-IDENTICAL DISTRIBUTED RANDOM VARIABLES UNDER SUBLINEAR EXPECTATIONS
}

\author{
MIAOMIAO GAO ${ }^{1}$, FENG HU ${ }^{2}$, AND ZHAOJUN ZONG ${ }^{3}$ \\ School of Statistics \\ Qufu Normal University \\ Qufu, 273165, P.R. CHINA
}

\begin{abstract}
In this paper, we study some limit theorems for random variables under sublinear expectations. First, a law of large numbers is proved for independent and non-identical distributed random variables with only finite first order moments. Second, a central limit theorem is proved for independent and non-identical distributed random variables with only finite second order moments. These results include and extend some existing results.
\end{abstract}

AMS Subject Classification: $60 \mathrm{H} 10,60 \mathrm{G} 48$

Received: February 3,2017; Accepted: October 2, 2017;

Published: March 11, $2018 \quad$ doi: 10.12732/dsa.v27i2.3

Dynamic Publishers, Inc., Acad. Publishers, Ltd. https://acadsol.eu/dsa

\section{INTRODUCTION}

The law of large numbers and central limit theorem as fundamental limit theorems in probability theory play a fruitful role in the development of probability theory and its applications. However, these kinds of limit theorems have always considered additive probabilities and additive expectations. In fact, the additivity of probabilities and expectations has been abandoned in some areas because many uncertain phenomena cannot be well modeled by using additive probabilities and additive expectations.

Since the paper (Artzner et al. [1]) on coherent risk measures, people are more and more interested in sublinear expectations (or more generally, convex expectations, see $[4,6,7,8])$. By Peng [16], we know that a sublinear expectation $\hat{E}$ can be 
represented as the upper expectation of a subset of linear expectations $\left\{E_{\theta}: \theta \in \Theta\right\}$, i.e., $\hat{E}[\cdot]=\sup _{\theta \in \Theta} E_{\theta}[\cdot]$. In most cases, this subset is often treated as an uncertain model of probabilities $\left\{P_{\theta}: \theta \in \Theta\right\}$ and the notion of sublinear expectation provides a robust way to measure a risk loss $X$. In fact, the nonlinear expectation theory provides many rich, flexible and elegant tools.

Since the notion of independent identically distributed (IID) random variables under sublinear expectations initiated by Peng, many limit results such as strong (weak) law of large numbers, central limit theorem and law of iterated logarithm under sublinear expectations have been studied. For more details, we can see $[2,3,9$, $10,11,12,13,14,15,18,19]$.

In this paper, we study some limit theorems for random variables under sublinear expectations. First, a law of large numbers is proved for independent and non-identical distributed random variables with only finite first order moments. Second, a central limit theorem is proved for independent and non-identical distributed random variables with only finite second order moments. These results generalize the known results in $[10,12,13,14,15,19]$.

\section{PRELIMINARIES}

In this section, we present some preliminaries in the theory of sublinear expectations. For more detail, we can see $[5,16,17,18,19]$.

Let $(\Omega, \mathcal{F})$ be a given measurable space and let $\mathcal{H}$ be a linear space of real functions defined on $(\Omega, \mathcal{F})$. We suppose that $\mathcal{H}$ satisfies $c \in \mathcal{H}$ for each constant $c$ and $|X| \in \mathcal{H}$ if $X \in \mathcal{H}$. The space $\mathcal{H}$ can be considered as the space of random variables.

Definition 2.1. (see $[16,17,18,19]$ ). A sublinear expectation $\hat{E}$ on $\mathcal{H}$ is a functional $\hat{E}: \mathcal{H} \rightarrow \overline{\mathbb{R}}$ satisfying the following properties: for all $X, Y \in \mathcal{H}$, we have

(a) Monotonicity: If $X \geq Y$, then $\hat{E}[X] \geq \hat{E}[Y]$;

(b) Constant preserving: $\hat{E}[c]=c, \forall c \in \mathbb{R}$;

(c) Sub-additivity: $\hat{E}[X+Y] \leq \hat{E}[X]+\hat{E}[Y]$ whenever $\hat{E}[X]+\hat{E}[Y]$ is not of the form $+\infty-\infty$ or $-\infty+\infty$;

(d) Positive homogeneity: $\hat{E}[\lambda X]=\lambda \hat{E}[X], \forall \lambda \geq 0$.

Here $\overline{\mathbb{R}}=[-\infty,+\infty]$. The triple $(\Omega, \mathcal{H}, \hat{E})$ is called a sublinear expectation space.

Give a sublinear expectation $\hat{E}$, let us denote the conjugate expectation $\hat{\varepsilon}$ of $\hat{E}$ by

$$
\hat{\varepsilon}[X]:=-\hat{E}[-X], \quad \forall X \in \mathcal{H}
$$

It is obvious that $\hat{\varepsilon}[X] \leq \hat{E}[X]$, for all $X \in \mathcal{H}$. 
In this paper, we consider the following sublinear expectation space $(\Omega, \mathcal{H}, \hat{E})$ : if $X_{1}, \cdots, X_{n} \in \mathcal{H}$, then $\varphi\left(X_{1}, \cdots, X_{n}\right) \in \mathcal{H}$ for each $\varphi \in C_{\text {l.Lip }}\left(\mathbb{R}^{n}\right)$, where $C_{\text {l.Lip }}\left(\mathbb{R}^{n}\right)$ denotes the linear space of functions $\varphi$ satisfying

$$
|\varphi(x)-\varphi(y)| \leq C\left(1+|x|^{m}+|y|^{m}\right)|x-y| \quad \forall x, y \in \mathbb{R}^{n}
$$

for some $C>0, m \in \mathbb{N}$ depending on $\varphi$. Let $C_{b . \text { Lip }}\left(\mathbb{R}^{n}\right)$ denote the linear space of bounded functions $\varphi$ satisfying

$$
|\varphi(x)-\varphi(y)| \leq C|x-y| \quad \forall x, y \in \mathbb{R}^{n}
$$

for some $C>0$ depending on $\varphi$.

Definition 2.2. (1See $[16,17,18,19]$ ). Identical distribution: Let $X_{1}$ and $X_{2}$ be two n-dimensional random vectors defined in sublinear expectation spaces $\left(\Omega_{1}, \mathcal{H}_{1}, \hat{E}_{1}\right)$ and $\left(\Omega_{2}, \mathcal{H}_{2}, \hat{E}_{2}\right)$, respectively. They are called identically distributed, denoted by $X_{1} \stackrel{d}{=} X_{2}$, if

$$
\hat{E}_{1}\left[\varphi\left(X_{1}\right)\right]=\hat{E}_{2}\left[\varphi\left(X_{2}\right)\right], \quad \forall \varphi \in C_{l . L i p}\left(\mathbb{R}^{n}\right),
$$

whenever the sublinear expectations are finite.

Independence: In a sublinear expectation space $(\Omega, \mathcal{H}, \hat{E})$, a random vector $Y=$ $\left(Y_{1}, \cdots, Y_{n}\right), Y_{i} \in \mathcal{H}$ is called independent to another random vector $X:=\left(X_{1}, \cdots\right.$, $\left.X_{m}\right), X_{i} \in \mathcal{H}$ under $\hat{E}$, if for each test function $\varphi \in C_{\text {l.Lip }}\left(\mathbb{R}^{m} \times \mathbb{R}^{n}\right)$, we have

$$
\hat{E}[\varphi(X, Y)]=\hat{E}\left[\hat{E}[\varphi(x, Y)]_{x=X}\right]
$$

whenever $\bar{\varphi}(x):=\hat{E}[|\varphi(x, Y)|]<\infty$ for all $x$ and $\hat{E}[\bar{\varphi}(X)]<\infty$.

Sequence of IID random variables: A sequence of IID random sequence $\left\{X_{i}\right\}_{i=1}^{\infty}$ is called IID random variables, if $X_{i} \stackrel{d}{=} X_{1}$ and $X_{i+1}$ is independent to $Y:=\left(X_{1}, \cdots, X_{i}\right)$ for each $i \geq 1$.

Definition 2.3. (Maximal distribution) (see $[16,17]$ ). A random variable $\eta$ in a sublinear expectation space $(\Omega, \mathcal{H}, \tilde{E})$ is called maximal distributed if

$$
\tilde{E}[\varphi(\eta)]=\sup _{\underline{\mu} \leq y \leq \bar{\mu}} \varphi(y), \quad \forall \varphi \in C_{l . L i p}(\mathbb{R}),
$$

where $\bar{\mu}:=\tilde{E}[\eta]$ and $\underline{\mu}:=\tilde{\varepsilon}[\eta]$.

Remark 2.4. Let $\eta$ be maximal distributed with $\bar{\mu}:=\tilde{E}[\eta], \underline{\mu}:=\tilde{\varepsilon}[\eta]$, the distribution of $\eta$ is characterized by the following parabolic partial differential equation (PDE):

$$
\partial_{t} u-g\left(\partial_{x} u\right)=0, \quad u(0, x)=\varphi(x),
$$

where $u(t, x):=\tilde{E}[\varphi(x+t \eta)],(t, x) \in[0, \infty) \times \mathbb{R}, g(x):=\bar{\mu} x^{+}-\underline{\mu} x^{-}$. 
Definition 2.5. (G-normal distribution) (see $[16,17])$. A random variable $X$ in a sublinear expectation space $(\Omega, \mathcal{H}, \tilde{E})$ with $\bar{\sigma}^{2}:=\tilde{E}\left[X^{2}\right], \underline{\sigma}^{2}:=\tilde{\varepsilon}\left[X^{2}\right]$ is called G-normal distributed, denoted by $X \sim \mathcal{N}\left(0 ;\left[\underline{\sigma}^{2}, \bar{\sigma}^{2}\right]\right)$, if for each $Y \in \mathcal{H}$ which is independent to $X$ such that $Y \stackrel{d}{=} X$, it holds that $a X+b Y \stackrel{d}{=} \sqrt{a^{2}+b^{2}} X, \forall a, b \geq 0$.

Remark 2.6. Let $X \sim \mathcal{N}\left(0 ;\left[\underline{\sigma}^{2}, \bar{\sigma}^{2}\right]\right)$ under $\tilde{E}$. For each $\varphi \in C_{l . L i p}(\mathbb{R})$, we define a function

$$
v(t, x):=\tilde{E}[\varphi(x+\sqrt{t} X)], \quad(t, x) \in[0, \infty) \times \mathbb{R} .
$$

Then $v$ is the unique viscosity solution of the following parabolic PDE:

$$
\partial_{t} v-G\left(\partial_{x x}^{2} v\right)=0, \quad v(0, x)=\varphi(x)
$$

where $G(\alpha):=\frac{1}{2} \tilde{E}\left[\alpha X^{2}\right]=\frac{1}{2}\left(\bar{\sigma}^{2} \alpha^{+}-\underline{\sigma}^{2} \alpha^{-}\right)$.

Lemma 2.7. (see $[3,5])$. Suppose $X \sim \mathcal{N}\left(0 ;\left[\underline{\sigma}^{2}, \bar{\sigma}^{2}\right]\right)$ under $\tilde{E}$. Let $P$ be a probability measure and $\varphi$ be a bounded continuous function on $\mathbb{R}$. If $\left\{B_{t}\right\}_{t \geq 0}$ is a 1dimensional Brownian motion under $P$, then

$$
\tilde{E}[\varphi(X)]=\sup _{\theta \in \Theta} E_{P}\left[\varphi\left(\int_{0}^{1} \theta_{s} d B_{s}\right)\right], \quad \tilde{\varepsilon}[\varphi(X)]=\inf _{\theta \in \Theta} E_{P}\left[\varphi\left(\int_{0}^{1} \theta_{s} d B_{s}\right)\right],
$$

where

$$
\begin{aligned}
& \Theta:=\left\{\left\{\theta_{t}\right\}_{t \geq 0}: \theta_{t} \text { is } \mathcal{F}_{t} \text {-adapted process such that } \underline{\sigma} \leq \theta_{t} \leq \bar{\sigma}\right\} \\
& \mathcal{F}_{t}:=\sigma\left\{B_{s}: 0 \leq s \leq t\right\} \vee \mathcal{N}, \mathcal{N} \text { is the collection of P-null subsets. }
\end{aligned}
$$

Lemma 2.8. (Hölder's inequality) (see [17]). Let $X, Y$ be two random variables in a sublinear expectation space $(\Omega, \mathcal{H}, \hat{E})$, then for $1<p, q<\infty, \frac{1}{p}+\frac{1}{q}=1$, we have

$$
\hat{E}[|X Y|] \leq\left(\hat{E}\left[|X|^{p}\right]\right)^{\frac{1}{p}} \cdot\left(\hat{E}\left[|Y|^{q}\right]\right)^{\frac{1}{q}} .
$$

Lemma 2.9. (Rosenthal's inequality) (see [18]). Let $\left(X_{1}, \cdots, X_{n}\right)$ be a sequence of random variables in $(\Omega, \mathcal{H}, \hat{E})$. Suppose that $X_{k+1}$ is independent to $\left(X_{1}, \cdots, X_{k}\right)$ for each $k=1, \cdots, n-1$. Denote $S_{n}:=\sum_{k=1}^{n} X_{k}$.

(a) Then

$$
\begin{aligned}
\hat{E}\left[\max _{k \leq n}\left|S_{k}\right|^{p}\right] \leq & C_{p}\left\{\sum_{k=1}^{n} \hat{E}\left[\left|X_{k}\right|^{p}\right]+\left(\sum_{k=1}^{n} \hat{E}\left[X_{k}^{2}\right]\right)^{\frac{p}{2}}\right\} \\
& +C_{p}\left\{\left(\sum_{k=1}^{n}\left[\left(\hat{\varepsilon}\left[X_{k}\right]\right)^{-}+\left(\hat{E}\left[X_{k}\right]\right)^{+}\right]\right)^{p}\right\}, \quad \text { for } p \geq 2 .
\end{aligned}
$$


(b) Furthermore, we assume that $\hat{E}\left[X_{k}\right] \leq 0, \quad k=1, \cdots, n$. Then

$$
\hat{E}\left[\left(S_{n}^{+}\right)^{p}\right] \leq \begin{cases}2^{2-p} \sum_{k=1}^{n} \hat{E}\left[\left|X_{k}\right|^{p}\right], & \text { for } 1 \leq p \leq 2, \\ C_{p} n^{\frac{p}{2}-1} \sum_{k=1}^{n} \hat{E}\left[\left|X_{k}\right|^{p}\right], & \text { for } p \geq 2 .\end{cases}
$$

Definition 2.10. A set function $V: \mathcal{F} \rightarrow[0,1]$ is called a capacity if it satisfies the following:

(1) $V(\emptyset)=0, V(\Omega)=1$;

(2) $V(A) \leq V(B)$, whenever $A \subset B$ and $A, B \in \mathcal{F}$.

It is called a sub-additive capacity if it further satisfies $V(A \cup B) \leq V(A)+V(B)$ for all $A, B \in \mathcal{F}$ with $A \cup B \in \mathcal{F}$.

Let $(\Omega, \mathcal{H}, \hat{E})$ be a sublinear expectation space, and $\hat{\varepsilon}$ be the conjugate expectation of $\hat{E}$. We denote a pair $(\mathbb{V}, v)$ of capacities by

$$
\mathbb{V}(A):=\inf \left\{\hat{E}[\xi]: I_{A} \leq \xi, \xi \in \mathcal{H}\right\}, \quad v(A):=1-\mathbb{V}\left(A^{c}\right), \quad \forall A \in \mathcal{F},
$$

where $A^{c}$ is the complement set of $A$. Then

$$
\begin{aligned}
& \mathbb{V}(A):=\hat{E}\left[I_{A}\right], \quad v(A):=\hat{\varepsilon}\left[I_{A}\right], \quad \text { if } I_{A} \in \mathcal{H}, \\
& \hat{E}[f] \leq \mathbb{V}(A) \leq \hat{E}[g], \quad \hat{\varepsilon}[f] \leq v(A) \leq \hat{\varepsilon}[g], \quad \text { if } f \leq I_{A} \leq g, \quad f, g \in \mathcal{H} .
\end{aligned}
$$

Obviously, $\mathbb{V}$ is sub-additive. But $v$ is not. However, we have $v(A \cup B) \leq v(A)+\mathbb{V}(B)$.

\section{MAIN RESULTS}

Theorem 3.1. Let a sequence $\left\{X_{i}\right\}_{i=1}^{\infty}$, which is in a sublinear expectation space $(\Omega, \mathcal{H}, \hat{E})$, satisfy the following conditions:

(i) each $X_{i+1}$ is independent to $\left(X_{1}, \cdots, X_{i}\right)$, for $i=1,2, \cdots$;

(ii) $\hat{E}\left[X_{i}\right]=\overline{\mu_{i}}, \hat{\varepsilon}\left[X_{i}\right]=\underline{\mu_{i}}$, where $-\infty<\underline{\mu_{i}} \leq \overline{\mu_{i}}<\infty$;

(iii) there are two constants $\bar{\mu}$ and $\underline{\mu}$ such that

$$
\lim _{n \rightarrow \infty} \frac{1}{n} \sum_{i=1}^{n}\left|\underline{\mu_{i}}-\underline{\mu}\right|=0, \quad \lim _{n \rightarrow \infty} \frac{1}{n} \sum_{i=1}^{n}\left|\overline{\mu_{i}}-\bar{\mu}\right|=0 ;
$$

(iv) $\lim _{d \rightarrow \infty} \sup _{i \geq 1} \hat{E}\left[\left(\left|X_{i}\right|-d\right)^{+}\right]=0$;

(v) $\sup _{i \geq 1} \hat{E}\left[\left|X_{i}\right|\right]<\infty$. Then for any continuous function $\varphi$ satisfying $|\varphi(x)| \leq$ $C(1+|x|)$, we have

$$
\lim _{n \rightarrow \infty} \hat{E}\left[\varphi\left(\frac{S_{n}}{n}\right)\right]=\widetilde{E}[\varphi(\eta)]
$$


where $S_{n}=\sum_{i=1}^{n} X_{i}, \eta$ is maximal distributed under $\widetilde{E}$ with $\bar{\mu}:=\widetilde{E}[\eta], \underline{\mu}:=\widetilde{\varepsilon}[\eta]$. Furthermore, if $p>1$ and $\sup \hat{E}\left[\left|X_{i}\right|^{p}\right]<\infty$, then (3.1) holds for any continuous function $\varphi$ satisfying $|\varphi(x)| \leq C\left(1+|x|^{p}\right)$.

Proof. Let $Y_{i}=(-i) \vee\left(X_{i} \wedge i\right), T_{n}=\sum_{i=1}^{n} Y_{i}$. In order to prove Theorem 3.1, we need the following facts:

(A1) Suppose that the condition (iv) is satisfied, then

$$
\frac{1}{n} \sum_{i=1}^{n} \hat{E}\left[\left|X_{i}-Y_{i}\right|\right] \rightarrow 0 \quad \text { as } \quad n \rightarrow \infty .
$$

(A2) Suppose that the conditions (iv) and (v) are satisfied, then

$$
\frac{\sum_{i=1}^{n} \hat{E}\left[\left|Y_{i}\right|^{\alpha+1}\right]}{n^{\alpha+1}} \rightarrow 0 \quad \text { as } \quad n \rightarrow \infty, \quad \forall 0<\alpha<1 .
$$

(A3) Suppose that the conditions (i) and (v) are satisfied, then

$$
\hat{E}\left[\left|T_{n}\right|^{2 p}\right] \leq C_{2 p} n^{2 p}, \quad \forall p \geq 1 .
$$

For (A1), by Stolz theorem, it is sufficient to show that

$$
\hat{E}\left[\left|X_{n}-Y_{n}\right|\right] \rightarrow 0 \quad \text { as } \quad n \rightarrow \infty .
$$

Note that

$$
\hat{E}\left[\left|X_{n}-Y_{n}\right|\right]=\hat{E}\left[\left(\left|X_{n}\right|-n\right)^{+}\right] \leq \sup _{i \geq 1} \hat{E}\left[\left(\left|X_{i}\right|-n\right)^{+}\right] \rightarrow 0 \quad \text { as } \quad n \rightarrow \infty .
$$

So (A1) holds.

For (A2), by Stolz theorem, it is sufficient to show that

$$
\frac{\hat{E}\left[\left|Y_{n}\right|^{\alpha+1}\right]}{n^{\alpha+1}-(n-1)^{\alpha+1}} \rightarrow 0 \quad \text { as } \quad n \rightarrow \infty .
$$

Note that

$$
\begin{aligned}
& \frac{\hat{E}\left[\left|Y_{n}\right|^{\alpha+1}\right]}{n^{\alpha+1}-(n-1)^{\alpha+1}} \leq \frac{\hat{E}\left[\left|Y_{n}\right|^{\alpha+1}\right]}{(n-1)^{\alpha}} \\
\hat{E}\left[\left|Y_{n}\right|^{\alpha+1}\right] & \leq \hat{E}\left[\left|X_{n}\right|\left|Y_{n}\right|^{\alpha}\right] \leq \hat{E}\left[\left(\left|X_{n}\right|-d+d\right)\left|Y_{n}\right|^{\alpha}\right] \\
& \leq n^{\alpha} \hat{E}\left[\left(\left|X_{n}\right|-d\right)^{+}\right]+d \hat{E}\left[\left|Y_{n}\right|^{\alpha}\right] \\
& \leq n^{\alpha} \hat{E}\left[\left(\left|X_{n}\right|-d\right)^{+}\right]+d\left(\hat{E}\left[\left|X_{n}\right|\right]\right)^{\alpha} \\
& \leq n^{\alpha} \cdot \sup _{i \geq 1} \hat{E}\left[\left(\left|X_{i}\right|-d\right)^{+}\right]+d\left(\sup _{i \geq 1} \hat{E}\left[\left|X_{i}\right|\right]\right)^{\alpha}
\end{aligned}
$$


So

$$
\begin{aligned}
& \frac{\hat{E}\left[\left|Y_{n}\right|^{\alpha+1}\right]}{(n-1)^{\alpha}} \leq \frac{n^{\alpha} \cdot \sup _{i \geq 1} \hat{E}\left[\left(\left|X_{i}\right|-d\right)^{+}\right]}{(n-1)^{\alpha}}+\frac{d\left(\sup _{i \geq 1} \hat{E}\left[\left|X_{i}\right|\right]\right)^{\alpha}}{(n-1)^{\alpha}} \rightarrow 0 \\
& \text { as } n \rightarrow \infty \text { and } d \rightarrow \infty \text {. }
\end{aligned}
$$

Thus, (A.2) holds.

For (A3), by the Rosenthal's inequality (2.1), we have

$$
\begin{aligned}
\hat{E}\left[\left|T_{n}\right|^{2 p}\right] & \leq C_{2 p} \sum_{i=1}^{n} \hat{E}\left[\left|Y_{i}\right|^{2 p}\right]+C_{2 p}\left(\sum_{i=1}^{n} \hat{E}\left[\left|Y_{i}\right|^{2}\right]\right)^{p} \\
& +C_{2 p}\left(\sum_{i=1}^{n}\left[\left(\hat{E}\left[Y_{i}\right]\right)^{+}+\left(\hat{E}\left[-Y_{i}\right]\right)^{+}\right]\right)^{2 p} \\
& \leq C_{2 p} \sum_{i=1}^{n} \hat{E}\left[\left|X_{i}\right|\left|Y_{i}\right|^{2 p-1}\right]+C_{2 p}\left(\sum_{i=1}^{n} \hat{E}\left[\left|X_{i}\right|\left|Y_{i}\right|\right]\right)^{p} \\
& +C_{2 p}\left(\sum_{i=1}^{n} 2 \hat{E}\left[\left|X_{i}\right|\right]\right)^{2 p} \\
& \leq C_{2 p} n^{2 p-1} n \cdot \sup _{i \geq 1} \hat{E}\left[\left|X_{i}\right|\right]+C_{2 p}\left(n \cdot n \cdot \sup _{i \geq 1} \hat{E}\left[\left|X_{i}\right|\right]\right)^{p} \\
& +C_{2 p} 2^{2 p}\left(n \cdot \sup _{i \geq 1} \hat{E}\left[\left|X_{i}\right|\right]\right)^{2 p} \\
& \leq C_{2 p} n^{2 p} .
\end{aligned}
$$

We first prove that

$$
\lim _{n \rightarrow \infty} \hat{E}\left[\varphi\left(\frac{T_{n}}{n}\right)\right]=\widetilde{E}[\varphi(\eta)], \quad \forall \varphi \in C_{b . L i p}(\mathbb{R})
$$

Now, for a small but fixed $h>0$, let $V$ be the unique viscosity solution of the following equation:

$$
\partial_{t} V+g\left(\partial_{x} V\right)=0, \quad(t, x) \in[0,1+h] \times \mathbb{R},\left.\quad V\right|_{t=1+h}=\varphi(x),
$$

where $g(x):=\bar{\mu} x^{+}-\underline{\mu} x^{-}$. According to the definition of maximal distribution, we have

$$
V(t, x)=\widetilde{E}[\varphi(x+(1+h-t) \eta)], \quad V(h, 0)=\widetilde{E}[\varphi(\eta)], \quad V(1+h, x)=\varphi(x) .
$$

Since (3.3) is a uniformly parabolic PDE, by the interior regularity of $V$, we have

$$
\|V\|_{C^{1+\frac{\alpha}{2}, 1+\alpha}([0,1] \times \mathbb{R})}<\infty, \text { for some } \alpha \in(0,1) .
$$


Let $\delta=\frac{1}{n}, T_{0}=0$, then

$$
\begin{aligned}
V\left(1, \delta T_{n}\right)-V(0,0)= & \sum_{i=0}^{n-1}\left\{V\left((i+1) \delta, \delta T_{i+1}\right)-V\left(i \delta, \delta T_{i}\right)\right\} \\
= & \sum_{i=0}^{n-1}\left\{\left[V\left((i+1) \delta, \delta T_{i+1}\right)-V\left(i \delta, \delta T_{i+1}\right)\right]\right. \\
& \left.+\left[V\left(i \delta, \delta T_{i+1}\right)-V\left(i \delta, \delta T_{i}\right)\right]\right\} \\
& =\sum_{i=0}^{n-1}\left\{I_{\delta}^{i}+J_{\delta}^{i}\right\}
\end{aligned}
$$

with, by Taylor's expansion,

$$
\begin{aligned}
& J_{\delta}^{i}=\partial_{t} V\left(i \delta, \delta T_{i}\right) \delta+\partial_{x} V\left(i \delta, \delta T_{i}\right) Y_{i+1} \delta \\
& =\left(\partial_{t} V\left(i \delta, \delta T_{i}\right) \delta+\partial_{x} V\left(i \delta, \delta T_{i}\right) X_{i+1} \delta\right)+\left(\partial_{x} V\left(i \delta, \delta T_{i}\right)\left(Y_{i+1}-X_{i+1}\right) \delta\right) \\
& =J_{\delta, 1}^{i}+J_{\delta, 2}^{i}, \\
& I_{\delta}^{i}=\int_{0}^{1}\left[\partial_{t} V\left((i+\beta) \delta, \delta T_{i+1}\right)-\partial_{t} V\left(i \delta, \delta T_{i+1}\right)\right] d \beta \delta+\left[\partial_{t} V\left(i \delta, \delta T_{i+1}\right)-\partial_{t} V\left(i \delta, \delta T_{i}\right)\right] \delta \\
& +\int_{0}^{1}\left[\partial_{x} V\left(i \delta, \delta T_{i}+\beta \delta Y_{i+1}\right)-\partial_{x} V\left(i \delta, \delta T_{i}\right)\right] d \beta Y_{i+1} \delta .
\end{aligned}
$$

Therefore,

$$
\begin{aligned}
& \hat{E}\left[\sum_{i=0}^{n-1} J_{\delta, 1}^{i}\right]-\sum_{i=0}^{n-1}\left(\hat{E}\left[\left|J_{\delta, 2}^{i}\right|\right]+\hat{E}\left[\left|I_{\delta}^{i}\right|\right]\right) \\
& \leq \hat{E}\left[V\left(1, \delta T_{n}\right)\right]-V(0,0) \leq \hat{E}\left[\sum_{i=0}^{n-1} J_{\delta, 1}^{i}\right]+\sum_{i=0}^{n-1}\left(\hat{E}\left[\left|J_{\delta, 2}^{i}\right|\right]+\hat{E}\left[\left|I_{\delta}^{i}\right|\right]\right) .
\end{aligned}
$$

We first consider $\hat{E}\left[\sum_{i=0}^{n-1} J_{\delta, 1}^{i}\right]$. From (3.3) and the condition (i), it follows that

$$
\begin{aligned}
\hat{E}\left[J_{\delta, 1}^{i}\right]= & \hat{E}\left[\partial_{t} V\left(i \delta, \delta T_{i}\right) \delta+\partial_{x} V\left(i \delta, \delta T_{i}\right) X_{i+1} \delta\right] \\
= & \hat{E}\left\{\partial_{t} V\left(i \delta, \delta T_{i}\right) \delta+\delta\left[\left(\partial_{x} V\left(i \delta, \delta T_{i}\right)\right)^{+} \overline{\mu_{i+1}}-\left(\partial_{x} V\left(i \delta, \delta T_{i}\right)\right)^{-} \underline{\mu_{i+1}}\right]\right\} \\
\leq & \hat{E}\left\{\partial_{t} V\left(i \delta, \delta T_{i}\right) \delta+\delta\left[\left(\partial_{x} V\left(i \delta, \delta T_{i}\right)\right)^{+} \bar{\mu}-\left(\partial_{x} V\left(i \delta, \delta T_{i}\right)\right)^{-} \underline{\mu}\right]\right\} \\
& +\delta \hat{E}\left[\left(\partial_{x} V\left(i \delta, \delta T_{i}\right)\right)^{+}\left(\overline{\mu_{i+1}}-\bar{\mu}\right)-\left(\partial_{x} V\left(i \delta, \delta T_{i}\right)\right)^{-}\left(\underline{\mu_{i+1}}-\underline{\mu}\right)\right] \\
= & \delta \hat{E}\left[\left(\partial_{x} V\left(i \delta, \delta T_{i}\right)\right)^{+}\left(\overline{\mu_{i+1}}-\bar{\mu}\right)-\left(\partial_{x} V\left(i \delta, \delta T_{i}\right)\right)^{-}\left(\underline{\mu_{i+1}}-\underline{\mu}\right)\right] \\
\leq & \delta \hat{E}\left[\left|\partial_{x} V\left(i \delta, \delta T_{i}\right)\right|\right]\left(\left|\overline{\mu_{i+1}}-\bar{\mu}\right|+\left|\underline{\mu_{i+1}}-\underline{\mu}\right|\right) .
\end{aligned}
$$


Since $\partial_{x} V$ is uniformly $\frac{\alpha}{2}$-hölder continuous in $t$ and $\alpha$-hölder continuous in $x$ on $[0,1] \times \mathbb{R}$, we have

$$
\begin{aligned}
\hat{E}\left[\left|\partial_{x} V\left(i \delta, \delta T_{i}\right)\right|\right] & \leq \hat{E}\left[\left|\partial_{x} V\left(i \delta, \delta T_{i}\right)-\partial_{x} V(0,0)\right|\right]+\left|\partial_{x} V(0,0)\right| \\
& \leq C\left(1+|i \delta|^{\frac{\alpha}{2}}+\hat{E}\left[\left|\delta T_{i}\right|^{\alpha}\right]\right) .
\end{aligned}
$$

Since

$$
\hat{E}\left[\left|\delta T_{i}\right|^{\alpha}\right] \leq \hat{E}\left[\left|\delta T_{i}\right|\right]+1 \leq \sup _{i \geq 1} \hat{E}\left[\left|X_{i}\right|\right]+1,
$$

we claim that there is a constant $C_{1}>0$, such that

$$
\hat{E}\left[\left|\partial_{x} V\left(i \delta, \delta T_{i}\right)\right|\right] \leq C_{1} .
$$

Then we obtain

$$
\hat{E}\left[\sum_{i=0}^{n-1} J_{\delta, 1}^{i}\right] \leq \sum_{i=0}^{n-1} \hat{E}\left[J_{\delta, 1}^{i}\right] \leq C_{1} \frac{1}{n} \sum_{i=0}^{n-1}\left(\left|\overline{\mu_{i+1}}-\bar{\mu}\right|+\left|\underline{\mu_{i+1}}-\underline{\mu}\right|\right) .
$$

In a similar manner as above, we also have

$$
\hat{E}\left[\sum_{i=0}^{n-1} J_{\delta, 1}^{i}\right] \geq-C_{1} \frac{1}{n} \sum_{i=0}^{n-1}\left(\left|\overline{\mu_{i+1}}-\bar{\mu}\right|+\left|\underline{\mu_{i+1}}-\underline{\mu}\right|\right) .
$$

Thus, from the condition (iii), we can obtain

$$
\lim _{n \rightarrow \infty} \hat{E}\left[\sum_{i=0}^{n-1} J_{\delta, 1}^{i}\right]=0
$$

Next, we consider $\sum_{i=0}^{n-1} \hat{E}\left[\left|J_{\delta, 2}^{i}\right|\right]$. According to the condition (i) and (3.6), we have

$$
\begin{aligned}
\hat{E}\left[\left|J_{\delta, 2}^{i}\right|\right] & =\hat{E}\left[\left|\partial_{x} V\left(i \delta, \delta T_{i}\right)\left(Y_{i+1}-X_{i+1}\right) \delta\right|\right] \\
& \leq \delta \hat{E}\left[\left|\partial_{x} V\left(i \delta, \delta T_{i}\right)\right|\right] \hat{E}\left[\left|X_{i+1}-Y_{i+1}\right|\right] \\
& \leq \delta C_{1} \hat{E}\left[\left|X_{i+1}-Y_{i+1}\right|\right] .
\end{aligned}
$$

Then, we obtain

$$
\sum_{i=0}^{n-1} \hat{E}\left[\left|J_{\delta, 2}^{i}\right|\right] \leq C_{1} \frac{1}{n} \sum_{i=0}^{n-1} \hat{E}\left[\left|X_{i+1}-Y_{i+1}\right|\right] .
$$

By (A1), it follows that

$$
\lim _{n \rightarrow \infty} \sum_{i=0}^{n-1} \hat{E}\left[\left|J_{\delta, 2}^{i}\right|\right]=0
$$

Finally, we consider $\sum_{i=0}^{n-1} \hat{E}\left[\left|I_{\delta}^{i}\right|\right]$. For $I_{\delta}^{i}$, since both $\partial_{t} V$ and $\partial_{x} V$ are uniformly $\frac{\alpha}{2}-$ hölder continuous in $t$ and $\alpha$-hölder continuous in $x$ on $[0,1] \times \mathbb{R}$, then we have

$$
\hat{E}\left[\left|I_{\delta}^{i}\right|\right] \leq C \delta^{\frac{\alpha}{2}+1}+C \delta^{\alpha+1}\left(\hat{E}\left[\left|Y_{i+1}\right|^{\alpha}\right]+\hat{E}\left[\left|Y_{i+1}\right|^{\alpha+1}\right]\right) .
$$


It follows that

$$
\sum_{i=0}^{n-1} \hat{E}\left[\left|I_{\delta}^{i}\right|\right] \leq C\left(\frac{1}{n}\right)^{\frac{\alpha}{2}}+C\left(\frac{1}{n}\right)^{\alpha+1} \sum_{i=0}^{n-1}\left(\hat{E}\left[\left|Y_{i+1}\right|^{\alpha}\right]+\hat{E}\left[\left|Y_{i+1}\right|^{\alpha+1}\right]\right) .
$$

For $\sum_{i=0}^{n-1} \hat{E}\left[\left|Y_{i+1}\right|^{\alpha}\right] / n^{\alpha+1}$, we have

$$
\frac{\sum_{i=0}^{n-1} \hat{E}\left[\left|Y_{i+1}\right|^{\alpha}\right]}{n^{\alpha+1}} \leq \frac{\sum_{i=0}^{n-1} \hat{E}\left[\left|X_{i+1}\right|^{\alpha}\right]}{n^{\alpha+1}} \leq \frac{\left(\sup _{i \geq 1} \hat{E}\left[\left|X_{i}\right|\right]\right)^{\alpha}}{n^{\alpha}} \rightarrow 0 \text { as } n \rightarrow \infty .
$$

By (A2), we can obtain

$$
\sum_{i=0}^{n-1} \hat{E}\left[\left|Y_{i+1}\right|^{\alpha+1}\right] / n^{\alpha+1} \rightarrow 0 \quad \text { as } \quad n \rightarrow \infty
$$

Therefore,

$$
\lim _{n \rightarrow \infty} \sum_{i=0}^{n-1} \hat{E}\left[\left|I_{\delta}^{i}\right|\right]=0
$$

From (3.5), (3.7), (3.8) and (3.9), we have

$$
\lim _{n \rightarrow \infty} \hat{E}\left[V\left(1, \delta T_{n}\right)\right]=V(0,0) .
$$

Additionally, it is obvious that if $\varphi \in C_{b . \text { Lip }}(\mathbb{R})$, i.e., $|\varphi(x)-\varphi(y)| \leq C|x-y|$, then for each $t, s \in[0,1+h]$ and $x \in \mathbb{R}$,

$$
|V(t, x)-V(s, x)| \leq C \widetilde{E}[|\eta|]|t-s| \leq C|t-s| .
$$

In particular,

$$
|V(0,0)-V(h, 0)| \leq C h .
$$

Combining (3.4), (3.11), with (3.12), we have

$$
\begin{aligned}
& \left|\hat{E}\left[\varphi\left(\delta T_{n}\right)\right]-\widetilde{E}[\varphi(\eta)]\right|=\left|\hat{E}\left[V\left(1+h, \delta T_{n}\right)\right]-V(h, 0)\right| \\
& \leq\left|\hat{E}\left[V\left(1+h, \delta T_{n}\right)\right]-\hat{E}\left[V\left(1, \delta T_{n}\right)\right]\right|+\left|\hat{E}\left[V\left(1, \delta T_{n}\right)\right]-V(0,0)\right|+|V(0,0)-V(h, 0)| \\
& \leq 2 C h+\left|\hat{E}\left[V\left(1, \delta T_{n}\right)\right]-V(0,0)\right| .
\end{aligned}
$$

From (3.10), we obtain

$$
\limsup _{n \rightarrow \infty}\left|\hat{E}\left[\varphi\left(\delta T_{n}\right)\right]-\widetilde{E}[\varphi(\eta)]\right| \leq 2 C h .
$$

So (3.2) is proved. 
By the Lipschitz continuity of $\varphi$ and (A1), we have

$$
\left|\hat{E}\left[\varphi\left(\frac{S_{n}}{n}\right)\right]-\hat{E}\left[\varphi\left(\frac{T_{n}}{n}\right)\right]\right| \leq C \frac{1}{n} \sum_{i=1}^{n} \hat{E}\left[\left|X_{i}-Y_{i}\right|\right] \rightarrow 0 \quad \text { as } \quad n \rightarrow \infty .
$$

Thus

$$
\lim _{n \rightarrow \infty} \hat{E}\left[\varphi\left(\frac{S_{n}}{n}\right)\right]=\widetilde{E}[\varphi(\eta)], \quad \forall \varphi \in C_{b . L i p}(\mathbb{R}) .
$$

If $\varphi$ is a bounded and uniformly continuous function, we can find a sequence $\left\{\varphi_{k}\right\}_{k=1}^{\infty} \in C_{b . L i p}(\mathbb{R})$ such that $\varphi_{k} \rightarrow \varphi$ uniformly on $\mathbb{R}$. By

$$
\begin{aligned}
& \left|\hat{E}\left[\varphi\left(\frac{S_{n}}{n}\right)\right]-\widetilde{E}[\varphi(\eta)]\right| \leq\left|\hat{E}\left[\varphi\left(\frac{S_{n}}{n}\right)\right]-\hat{E}\left[\varphi_{k}\left(\frac{S_{n}}{n}\right)\right]\right| \\
& +\left|\widetilde{E}[\varphi(\eta)]-\widetilde{E}\left[\varphi_{k}(\eta)\right]\right|+\left|\hat{E}\left[\varphi_{k}\left(\frac{S_{n}}{n}\right)\right]-\widetilde{E}\left[\varphi_{k}(\eta)\right]\right|,
\end{aligned}
$$

we can easily check that (3.1) holds.

Finally, suppose that $p \geq 1$, $\sup _{i \geq 1} \hat{E}\left[\left|X_{i}\right|^{p}\right]<\infty$, the conditions (i)-(iv) are satisfied, and $\varphi$ is a continuous function satisfying $|\varphi(x)| \leq C\left(1+|x|^{p}\right)$. Give a number $N>1$. Define $\varphi_{1}(x)=\varphi((-N) \vee(x \wedge N))$ and $\varphi_{2}(x)=\varphi(x)-\varphi_{1}(x)$. Then $\varphi_{1}$ is a bounded and uniformly continuous function and

$$
\left|\varphi_{2}(x)\right| \leq 4 C|x|^{p} I_{\{|x|>N\}} \leq 8 C\left(|x|^{p}-N / 2\right)^{+} .
$$

Define $M:=N / 2$, then $\left|\varphi_{2}(x)\right| \leq 8 C\left(|x|^{p}-M\right)^{+}$. So

$$
\begin{aligned}
& \left|\hat{E}\left[\varphi\left(\frac{S_{n}}{n}\right)\right]-\widetilde{E}[\varphi(\eta)]\right| \leq\left|\hat{E}\left[\varphi_{1}\left(\frac{S_{n}}{n}\right)\right]-\widetilde{E}\left[\varphi_{1}(\eta)\right]\right| \\
& +8 C \widetilde{E}\left[\left(|\eta|^{p}-M\right)^{+}\right]+8 C \hat{E}\left[\left(\left|\frac{S_{n}}{n}\right|^{p}-M\right)^{+}\right] .
\end{aligned}
$$

Since

$$
8 C \widetilde{E}\left[\left(|\eta|^{p}-M\right)^{+}\right] \leq 8 C \frac{\widetilde{E}\left[|\eta|^{2 p}\right]}{M} \rightarrow 0 \quad \text { as } \quad M \rightarrow \infty,
$$

then it is sufficient to show that

$$
\lim _{M \rightarrow \infty} \limsup _{n \rightarrow \infty} \hat{E}\left[\left(\left|\frac{S_{n}}{n}\right|^{p}-M\right)^{+}\right]=0 .
$$

Let $\hat{Y}_{i}=X_{i}-Y_{i}, \hat{S}_{n}=\sum_{i=1}^{n}\left(\hat{Y}_{i}-\hat{E}\left[\hat{Y}_{i}\right]\right)$, then

$$
S_{n}^{+} \leq T_{n}^{+}+\hat{S}_{n}^{+}+\sum_{i=1}^{n} \hat{E}\left[\left|\hat{Y}_{i}\right|\right]
$$




$$
\left(\left|\frac{S_{n}^{+}}{n}\right|^{p}-M\right)^{+} \leq\left(3^{p-1}\left|\frac{T_{n}^{+}}{n}\right|^{p}-M\right)^{+}+3^{p-1}\left|\frac{\hat{S}_{n}^{+}}{n}\right|^{p}+3^{p-1}\left(\sum_{i=1}^{n} \frac{\hat{E}\left[\left|\hat{Y}_{i}\right|\right]}{n}\right)^{p} .
$$

By (A1), we have

$$
\sum_{i=1}^{n} \frac{\hat{E}\left[\left|\hat{Y}_{i}\right|\right]}{n}=\frac{1}{n} \sum_{i=1}^{n} \hat{E}\left[\left|X_{i}-Y_{i}\right|\right] \rightarrow 0 \quad \text { as } \quad n \rightarrow \infty
$$

And by (A3), we have

$$
\hat{E}\left[\left(3^{p-1}\left|\frac{T_{n}^{+}}{n}\right|^{p}-M\right)^{+}\right] \leq M^{-1} 3^{2 p-2} \hat{E}\left[\left|\frac{T_{n}}{n}\right|^{2 p}\right] \leq M^{-1} 3^{2 p-2} C_{2 p} \rightarrow 0
$$

as $\quad M \rightarrow \infty$.

For $\hat{E}\left[\left|\hat{S}_{n}^{+} / n\right|^{p}\right]$, applying the Rosenthal's inequality (2.2), we can obtain the following result: When $p=1$,

$$
\hat{E}\left[\left|\frac{\hat{S}_{n}^{+}}{n}\right|\right] \leq 4 \frac{1}{n} \sum_{i=1}^{n} \hat{E}\left[\left|\hat{Y}_{i}\right|\right] \leq 4 \frac{1}{n} \sum_{i=1}^{n} \hat{E}\left[\left(\left|X_{i}\right|-i\right)^{+}\right] \leq 4 \frac{1}{n} \sum_{i=1}^{n} \sup _{k \geq 1} \hat{E}\left[\left(\left|X_{k}\right|-i\right)^{+}\right],
$$

so

$$
\hat{E}\left[\left|\frac{\hat{S}_{n}^{+}}{n}\right|\right] \rightarrow 0 \quad \text { as } \quad n \rightarrow \infty
$$

When $1<p \leq 2$,

$$
\begin{aligned}
\hat{E}\left[\left|\hat{S}_{n}^{+}\right|^{p}\right] & \leq 2^{2-p} \sum_{i=1}^{n} \hat{E}\left[\left|\hat{Y}_{i}-\hat{E}\left[\hat{Y}_{i}\right]\right|^{p}\right] \leq 2^{2-p} \sum_{i=1}^{n}\left(2^{p} \hat{E}\left[\left|\hat{Y}_{i}\right|^{p}\right]\right) \\
& \leq 4 \sum_{i=1}^{n} \hat{E}\left[\left|X_{i}\right|^{p}\right] \leq 4 n \cdot \sup _{i \geq 1} \hat{E}\left[\left|X_{i}\right|^{p}\right]
\end{aligned}
$$

so

$$
\hat{E}\left[\left|\frac{\hat{S}_{n}^{+}}{n}\right|^{p}\right] \rightarrow 0 \quad \text { as } \quad n \rightarrow \infty .
$$

When $p>2$,

$$
\begin{aligned}
\hat{E}\left[\left|\hat{S}_{n}^{+}\right|^{p}\right] & \leq C_{p} n^{\frac{p}{2}-1} \sum_{i=1}^{n} \hat{E}\left[\left|\hat{Y}_{i}-\hat{E}\left[\hat{Y}_{i}\right]\right|^{p}\right] \leq C_{p} n^{\frac{p}{2}-1} \sum_{i=1}^{n}\left(2^{p} \hat{E}\left[\left|\hat{Y}_{i}\right|^{p}\right]\right) \\
& \leq 2^{p} C_{p} n^{\frac{p}{2}-1} \sum_{i=1}^{n} \hat{E}\left[\left|X_{i}\right|^{p}\right] \leq 2^{p} C_{p}\left(\sup _{i \geq 1} \hat{E}\left[\left|X_{i}\right|^{p}\right]\right) n^{\frac{p}{2}}
\end{aligned}
$$

so

$$
\hat{E}\left[\left|\frac{\hat{S}_{n}^{+}}{n}\right|^{p}\right] \rightarrow 0 \quad \text { as } \quad n \rightarrow \infty .
$$


Thus, it follows that

$$
\lim _{M \rightarrow \infty} \limsup _{n \rightarrow \infty} \hat{E}\left[\left(\left|\frac{S_{n}^{+}}{n}\right|^{p}-M\right)^{+}\right]=0 .
$$

Similarly,

$$
\lim _{M \rightarrow \infty} \limsup _{n \rightarrow \infty} \hat{E}\left[\left(\left|\frac{S_{n}^{-}}{n}\right|^{p}-M\right)^{+}\right]=0 .
$$

So (3.13) is proved and the proof is completed.

Theorem 3.2. Under the conditions of Theorem 3.1, then for any $\varepsilon>0$,

$$
\lim _{n \rightarrow \infty} v\left(\frac{S_{n}}{n} \in(\underline{\mu}-\varepsilon, \bar{\mu}+\varepsilon)\right)=1,
$$

The proof is similar to the proof of Theorem 3.3 in [10] and so it is omitted.

Theorem 3.3. Let $\left\{X_{i}\right\}_{i=1}^{\infty}$ be a sequence of random variables in a sublinear expectation space $(\Omega, \mathcal{H}, \hat{E})$, satisfy the following conditions:

(i) each $X_{i+1}$ is independent to $\left(X_{1}, \cdots, X_{i}\right)$, for $i=1,2, \cdots$;

(ii) $\hat{E}\left[X_{i}\right]=\hat{\varepsilon}\left[X_{i}\right]=0, \hat{E}\left[X_{i}^{2}\right]=\bar{\sigma}_{i}^{2}, \hat{\varepsilon}\left[X_{i}^{2}\right]=\underline{\sigma}_{i}^{2}$, where $0 \leq \underline{\sigma}_{i} \leq \bar{\sigma}_{i}<\infty$;

(iii) there are two positive constants $\underline{\sigma}$ and $\bar{\sigma}$ such that

$$
\lim _{n \rightarrow \infty} \frac{1}{n} \sum_{i=1}^{n}\left|\underline{\sigma}_{i}^{2}-\underline{\sigma}^{2}\right|=0, \quad \lim _{n \rightarrow \infty} \frac{1}{n} \sum_{i=1}^{n}\left|\bar{\sigma}_{i}^{2}-\bar{\sigma}^{2}\right|=0 ;
$$

(iv) $\lim _{c \rightarrow \infty} \sup _{i \geq 1} \hat{E}\left[\left(X_{i}^{2}-c\right)^{+}\right]=0$;

(v) $\sup _{i \geq 1} \hat{E}\left[X_{i}^{2}\right]<\infty$. Then for any continuous function $\varphi$ satisfying $|\varphi(x)| \leq$ $C\left(1+x^{2}\right)$, we have

$$
\lim _{n \rightarrow \infty} \hat{E}\left[\varphi\left(\frac{S_{n}}{\sqrt{n}}\right)\right]=\widetilde{E}[\varphi(\xi)],
$$

where $S_{n}=\sum_{i=1}^{n} X_{i}, \xi \sim \mathcal{N}\left(0 ;\left[\underline{\sigma}^{2}, \bar{\sigma}^{2}\right]\right)$ under $\widetilde{E}$. Furthermore, if $p>2$ and $\sup _{i \geq 1} \hat{E}\left[\left|X_{i}\right|^{p}\right]$ $<\infty$, then $(3.15)$ holds for any continuous function $\varphi$ satisfying $|\varphi(x)| \leq \stackrel{i \geq 1}{C}\left(1+|x|^{p}\right)$.

Proof. Let $Y_{i}=(-\sqrt{i}) \vee\left(X_{i} \wedge \sqrt{i}\right), T_{n}=\sum_{i=1}^{n} Y_{i}$. In order to prove Theorem 3.3, we need the following facts:

(B1) Suppose that the condition (iv) is satisfied, then

$$
\frac{\sum_{i=1}^{n} \hat{E}\left[\left|X_{i}-Y_{i}\right|\right]}{\sqrt{n}} \rightarrow 0 \text { as } n \rightarrow \infty .
$$


(B2) Suppose that the conditions (iv) and (v) are satisfied, then

$$
\frac{\sum_{i=1}^{n} \hat{E}\left[\left|Y_{i}\right|^{\alpha+2}\right]}{n^{\frac{\alpha}{2}+1}} \rightarrow 0 \quad \text { as } \quad n \rightarrow \infty, \quad \forall 0<\alpha<1 .
$$

(B3) Suppose that the conditions (i), (ii), (iv) and (v) are satisfied, then

$$
\hat{E}\left[\left|T_{n}\right|^{p}\right] \leq C_{p} n^{\frac{p}{2}}, \quad \forall p \geq 2 .
$$

For (B1), note that

$$
\sqrt{n} \hat{E}\left[\left|X_{n}-Y_{n}\right|\right] \leq \hat{E}\left[\left(X_{n}^{2}-n\right)^{+}\right] \leq \sup _{i \geq 1} \hat{E}\left[\left(X_{i}^{2}-n\right)^{+}\right]
$$

So (B1) holds.

For (B2), note that

$$
\begin{aligned}
\hat{E}\left[\left|Y_{n}\right|^{\alpha+2}\right] & \leq \hat{E}\left[X_{n}^{2}\left|Y_{n}\right|^{\alpha}\right] \leq \hat{E}\left[\left(X_{n}^{2}-c+c\right)\left|Y_{n}\right|^{\alpha}\right] \\
& \leq n^{\frac{\alpha}{2}} \hat{E}\left[\left(X_{n}^{2}-c\right)^{+}\right]+c \hat{E}\left[\left|Y_{n}\right|^{\alpha}\right] \\
& \leq n^{\frac{\alpha}{2}} \hat{E}\left[\left(X_{n}^{2}-c\right)^{+}\right]+c\left(\hat{E}\left[X_{n}^{2}\right]\right)^{\frac{\alpha}{2}} \\
& \leq n^{\frac{\alpha}{2}} \cdot \sup _{i \geq 1} \hat{E}\left[\left(X_{i}^{2}-c\right)^{+}\right]+c\left(\sup _{i \geq 1} \hat{E}\left[X_{i}^{2}\right]\right)^{\frac{\alpha}{2}}
\end{aligned}
$$

for any $c>1$. By Stolz theorem, (B2) is true.

For (B3), by the Rosenthal's inequality (2.1) and (B1), we have

$$
\begin{aligned}
\hat{E}\left[\left|T_{n}\right|^{p}\right] & \leq C_{p} \sum_{i=1}^{n} \hat{E}\left[\left|Y_{i}\right|^{p}\right]+C_{p}\left(\sum_{i=1}^{n} \hat{E}\left[Y_{i}^{2}\right]\right)^{\frac{p}{2}}+C_{p}\left(\sum_{i=1}^{n}\left[\left(\hat{E}\left[Y_{i}\right]\right)^{+}+\left(\hat{E}\left[-Y_{i}\right]\right)^{+}\right]\right)^{p} \\
& \leq C_{p} n^{\frac{p}{2}-1} \sum_{i=1}^{n} \hat{E}\left[X_{i}^{2}\right]+C_{p}\left(\sum_{i=1}^{n} \hat{E}\left[X_{i}^{2}\right]\right)^{\frac{p}{2}}+C_{p}\left(\sum_{i=1}^{n} 2 \hat{E}\left[\left|X_{i}-Y_{i}\right|\right]\right)^{p} \\
& \leq C_{p} n^{\frac{p}{2}-1} \cdot n \cdot \sup _{i \geq 1} \hat{E}\left[X_{i}^{2}\right]+C_{p}\left(n \cdot \sup _{i \geq 1} \hat{E}\left[X_{i}^{2}\right]\right)^{\frac{p}{2}}+C_{p}\left(\sum_{i=1}^{n} 2 \hat{E}\left[\left|X_{i}-Y_{i}\right|\right]\right)^{p} \\
& \leq C_{p} n^{\frac{p}{2}}
\end{aligned}
$$

So (B3) is true.

First, we show that

$$
\lim _{n \rightarrow \infty} \hat{E}\left[\varphi\left(\frac{T_{n}}{\sqrt{n}}\right)\right]=\widetilde{E}[\varphi(\xi)], \quad \forall \varphi \in C_{b . L i p}(\mathbb{R}) .
$$


Now, for a small but fixed $h>0$, let $V$ be the unique viscosity solution of the following equation:

$$
\partial_{t} V+G\left(\partial_{x x}^{2} V\right)=0, \quad(t, x) \in[0,1+h] \times \mathbb{R},\left.\quad V\right|_{t=1+h}=\varphi(x),
$$

where $G(\alpha):=\frac{1}{2}\left(\bar{\sigma}^{2} \alpha^{+}-\underline{\sigma}^{2} \alpha^{-}\right)$. According to the definition of G-normal distribution, we have

$$
\begin{aligned}
V(t, x) & =\widetilde{E}[\varphi(x+\sqrt{1+h-t} \xi)], \\
V(h, 0) & =\widetilde{E}[\varphi(\xi)], \\
V(1+h, x) & =\varphi(x) .
\end{aligned}
$$

Since (3.17) is a uniformly parabolic PDE, by the interior regularity of $V$, we have

$$
\|V\|_{C^{1+\frac{\alpha}{2}, 2+\alpha}([0,1] \times \mathbb{R})}<\infty, \text { for some } \alpha \in(0,1) .
$$

Let $\delta=\frac{1}{n}, T_{0}=0$, then

$$
\begin{aligned}
V\left(1, \sqrt{\delta} T_{n}\right)-V(0,0)= & \sum_{i=0}^{n-1}\left\{V\left((i+1) \delta, \sqrt{\delta} T_{i+1}\right)-V\left(i \delta, \sqrt{\delta} T_{i}\right)\right\} \\
= & \sum_{i=0}^{n-1}\left\{\left[V\left((i+1) \delta, \sqrt{\delta} T_{i+1}\right)-V\left(i \delta, \sqrt{\delta} T_{i+1}\right)\right]\right. \\
& \left.+\left[V\left(i \delta, \sqrt{\delta} T_{i+1}\right)-V\left(i \delta, \sqrt{\delta} T_{i}\right)\right]\right\} \\
= & \sum_{i=0}^{n-1}\left\{I_{\delta}^{i}+J_{\delta}^{i}\right\}
\end{aligned}
$$

with, by Taylor's expansion,

$$
\begin{aligned}
J_{\delta}^{i}= & \partial_{t} V\left(i \delta, \sqrt{\delta} T_{i}\right) \delta+\frac{1}{2} \partial_{x x}^{2} V\left(i \delta, \sqrt{\delta} T_{i}\right) Y_{i+1}^{2} \delta+\partial_{x} V\left(i \delta, \sqrt{\delta} T_{i}\right) Y_{i+1} \sqrt{\delta} \\
= & \left(\partial_{t} V\left(i \delta, \sqrt{\delta} T_{i}\right) \delta+\frac{1}{2} \partial_{x x}^{2} V\left(i \delta, \sqrt{\delta} T_{i}\right) X_{i+1}^{2} \delta+\partial_{x} V\left(i \delta, \sqrt{\delta} T_{i}\right) X_{i+1} \sqrt{\delta}\right) \\
& +\left(\frac{1}{2} \partial_{x x}^{2} V\left(i \delta, \sqrt{\delta} T_{i}\right)\left(Y_{i+1}^{2}-X_{i+1}^{2}\right) \delta+\partial_{x} V\left(i \delta, \sqrt{\delta} T_{i}\right)\left(Y_{i+1}-X_{i+1}\right) \sqrt{\delta}\right) \\
= & J_{\delta, 1}^{i}+J_{\delta, 2}^{i}, \\
I_{\delta}^{i}= & \int_{0}^{1}\left[\partial_{t} V\left((i+\beta) \delta, \sqrt{\delta} T_{i+1}\right)-\partial_{t} V\left(i \delta, \sqrt{\delta} T_{i+1}\right)\right] d \beta \delta \\
& +\left[\partial_{t} V\left(i \delta, \sqrt{\delta} T_{i+1}\right)-\partial_{t} V\left(i \delta, \sqrt{\delta} T_{i}\right)\right] \delta \\
& +\int_{0}^{1} \int_{0}^{1}\left[\partial_{x x}^{2} V\left(i \delta, \sqrt{\delta} T_{i}+\gamma \beta Y_{i+1} \sqrt{\delta}\right)-\partial_{x x}^{2} V\left(i \delta, \sqrt{\delta} T_{i}\right)\right] \gamma d \beta d \gamma Y_{i+1}^{2} \delta .
\end{aligned}
$$


Therefore,

$$
\begin{aligned}
& \hat{E}\left[\sum_{i=0}^{n-1} J_{\delta, 1}^{i}\right]-\sum_{i=0}^{n-1}\left(\hat{E}\left[\left|J_{\delta, 2}^{i}\right|\right]+\hat{E}\left[\left|I_{\delta}^{i}\right|\right]\right) \leq \hat{E}\left[V\left(1, \sqrt{\delta} T_{n}\right)\right]-V(0,0) \\
& \leq \hat{E}\left[\sum_{i=0}^{n-1} J_{\delta, 1}^{i}\right]+\sum_{i=0}^{n-1}\left(\hat{E}\left[\left|J_{\delta, 2}^{i}\right|\right]+\hat{E}\left[\left|I_{\delta}^{i}\right|\right]\right) .
\end{aligned}
$$

For $I_{\delta}^{i}$, since both $\partial_{t} V$ and $\partial_{x x}^{2} V$ are uniformly $\frac{\alpha}{2}$-hölder continuous in $t$ and $\alpha$-hölder continuous in $x$ on $[0,1] \times \mathbb{R}$, then we have

$$
\left|I_{\delta}^{i}\right| \leq C \delta^{1+\frac{\alpha}{2}}\left(1+\left|Y_{i+1}\right|^{\alpha}+\left|Y_{i+1}\right|^{2+\alpha}\right)
$$

From (B2), we have

$$
\sum_{i=0}^{n-1} \hat{E}\left[\left|I_{\delta}^{i}\right|\right] \leq C\left(\frac{1}{n}\right)^{1+\frac{\alpha}{2}} \sum_{i=0}^{n-1}\left(1+\hat{E}\left[\left|Y_{i+1}\right|^{\alpha}\right]+\hat{E}\left[\left|Y_{i+1}\right|^{2+\alpha}\right]\right) \rightarrow 0 \quad \text { as } \quad n \rightarrow \infty .
$$

Thus

$$
\lim _{n \rightarrow \infty} \sum_{i=0}^{n-1} \hat{E}\left[\left|I_{\delta}^{i}\right|\right]=0
$$

For $J_{\delta, 1}^{i}$, from the conditions (i) and (ii) we have

$$
\hat{E}\left[\partial_{x} V\left(i \delta, \sqrt{\delta} T_{i}\right) X_{i+1} \sqrt{\delta}\right]=\hat{E}\left[-\partial_{x} V\left(i \delta, \sqrt{\delta} T_{i}\right) X_{i+1} \sqrt{\delta}\right]=0
$$

We then combine the above equality with (3.17) as well as the condition (i), it follows that

$$
\begin{aligned}
\hat{E}\left[J_{\delta, 1}^{i}\right]= & \hat{E}\left[\partial_{t} V\left(i \delta, \sqrt{\delta} T_{i}\right) \delta+\frac{1}{2} \partial_{x x}^{2} V\left(i \delta, \sqrt{\delta} T_{i}\right) X_{i+1}^{2} \delta\right] \\
= & \hat{E}\left\{\partial_{t} V\left(i \delta, \sqrt{\delta} T_{i}\right) \delta+\frac{\delta}{2}\left[\left(\partial_{x x}^{2} V\left(i \delta, \sqrt{\delta} T_{i}\right)\right)^{+} \bar{\sigma}_{i+1}^{2}-\left(\partial_{x x}^{2} V\left(i \delta, \sqrt{\delta} T_{i}\right)\right)^{-} \underline{\sigma}_{i+1}^{2}\right]\right\} \\
\leq & \hat{E}\left\{\partial_{t} V\left(i \delta, \sqrt{\delta} T_{i}\right) \delta+\frac{\delta}{2}\left[\left(\partial_{x x}^{2} V\left(i \delta, \sqrt{\delta} T_{i}\right)\right)^{+} \bar{\sigma}^{2}-\left(\partial_{x x}^{2} V\left(i \delta, \sqrt{\delta} T_{i}\right)\right)^{-} \underline{\sigma}^{2}\right]\right\} \\
& +\frac{\delta}{2} \hat{E}\left[\left(\partial_{x x}^{2} V\left(i \delta, \sqrt{\delta} T_{i}\right)\right)^{+}\left(\bar{\sigma}_{i+1}^{2}-\bar{\sigma}^{2}\right)-\left(\partial_{x x}^{2} V\left(i \delta, \sqrt{\delta} T_{i}\right)\right)^{-}\left(\underline{\sigma}_{i+1}^{2}-\underline{\sigma}^{2}\right)\right] \\
= & \frac{\delta}{2} \hat{E}\left[\left(\partial_{x x}^{2} V\left(i \delta, \sqrt{\delta} T_{i}\right)\right)^{+}\left(\bar{\sigma}_{i+1}^{2}-\bar{\sigma}^{2}\right)-\left(\partial_{x x}^{2} V\left(i \delta, \sqrt{\delta} T_{i}\right)\right)^{-}\left(\underline{\sigma}_{i+1}^{2}-\underline{\sigma}^{2}\right)\right] \\
\leq & \frac{\delta}{2} \hat{E}\left[\left|\partial_{x x}^{2} V\left(i \delta, \sqrt{\delta} T_{i}\right)\right|\right]\left(\left|\bar{\sigma}_{i+1}^{2}-\bar{\sigma}^{2}\right|+\left|\underline{\sigma}_{i+1}^{2}-\underline{\sigma}^{2}\right|\right) .
\end{aligned}
$$

Since $\partial_{x x}^{2} V$ is uniformly $\alpha$-hölder continuous in $x$ and $\frac{\alpha}{2}$-hölder continuous in $t$ on $[0,1] \times \mathbb{R}$, it follows that

$$
\hat{E}\left[\left|\partial_{x x}^{2} V\left(i \delta, \sqrt{\delta} T_{i}\right)\right|\right] \leq\left|\partial_{x x}^{2} V(0,0)\right|+\hat{E}\left[\left|\partial_{x x}^{2} V\left(i \delta, \sqrt{\delta} T_{i}\right)-\partial_{x x}^{2} V(0,0)\right|\right]
$$




$$
\begin{aligned}
& \leq C\left(1+|i \delta|^{\frac{\alpha}{2}}+\hat{E}\left[\left|\sqrt{\delta} T_{i}\right|^{\alpha}\right]\right) \\
& \leq C\left(1+|i \delta|^{\frac{\alpha}{2}}+\left(\hat{E}\left[\left|\sqrt{\delta} T_{i}\right|^{2}\right]\right)^{\frac{\alpha}{2}}\right) \leq C
\end{aligned}
$$

by Hölder's inequality and (B3). So we have

$$
\hat{E}\left[J_{\delta, 1}^{i}\right] \leq C \delta\left(\left|\bar{\sigma}_{i+1}^{2}-\bar{\sigma}^{2}\right|+\left|\underline{\sigma}_{i+1}^{2}-\underline{\sigma}^{2}\right|\right) .
$$

By the condition (iii), it follows that

$\hat{E}\left[\sum_{i=0}^{n-1} J_{\delta, 1}^{i}\right] \leq \sum_{i=0}^{n-1} \hat{E}\left[J_{\delta, 1}^{i}\right] \leq C \frac{1}{n} \sum_{i=0}^{n-1}\left(\left|\bar{\sigma}_{i+1}^{2}-\bar{\sigma}^{2}\right|+\left|\underline{\sigma}_{i+1}^{2}-\underline{\sigma}^{2}\right|\right) \rightarrow 0 \quad$ as $\quad n \rightarrow \infty$.

Similarly,

$$
\hat{E}\left[\sum_{i=0}^{n-1} J_{\delta, 1}^{i}\right] \geq-C \frac{1}{n} \sum_{i=0}^{n-1}\left(\left|\bar{\sigma}_{i+1}^{2}-\bar{\sigma}^{2}\right|+\left|\underline{\sigma}_{i+1}^{2}-\underline{\sigma}^{2}\right|\right) \rightarrow 0 \quad \text { as } \quad n \rightarrow \infty .
$$

Thus

$$
\lim _{n \rightarrow \infty} \hat{E}\left[\sum_{i=0}^{n-1} J_{\delta, 1}^{i}\right]=0
$$

For $J_{\delta, 2}^{i}$, in a similar manner as above, we have

$$
\hat{E}\left[\left|\partial_{x} V\left(i \delta, \sqrt{\delta} T_{i}\right)\right|\right] \leq C .
$$

By the conditions (i), (iv), (B1) and Stolz theorem,

$$
\begin{aligned}
\sum_{i=0}^{n-1} \hat{E}\left[\left|J_{\delta, 2}^{i}\right|\right] \leq & \sum_{i=0}^{n-1}\left\{\frac{1}{2} \hat{E}\left[\left|\partial_{x x}^{2} V\left(i \delta, \sqrt{\delta} T_{i}\right)\right|\right] \hat{E}\left[\left|X_{i+1}^{2}-Y_{i+1}^{2}\right|\right] \delta\right. \\
& \left.+\hat{E}\left[\left|\partial_{x} V\left(i \delta, \sqrt{\delta} T_{i}\right)\right|\right] \hat{E}\left[\left|X_{i+1}-Y_{i+1}\right|\right] \sqrt{\delta}\right\} \\
\leq & C \frac{1}{n} \sum_{i=0}^{n-1} \hat{E}\left[\left(X_{i+1}^{2}-(i+1)\right)^{+}\right]+C \frac{1}{\sqrt{n}} \sum_{i=0}^{n-1} \hat{E}\left[\left|X_{i+1}-Y_{i+1}\right|\right] \\
\leq & C \frac{1}{n} \sum_{i=0}^{n-1} \sup _{k \geq 1} \hat{E}\left[\left(X_{k}^{2}-(i+1)\right)^{+}\right]+C \frac{1}{\sqrt{n}} \sum_{i=1}^{n} \hat{E}\left[\left|X_{i}-Y_{i}\right|\right] \rightarrow 0 \\
& \text { as } \quad n \rightarrow \infty .
\end{aligned}
$$

Then combining (3.19), (3.20) and (3.21), it follows that

$$
\lim _{n \rightarrow \infty} \hat{E}\left[V\left(1, \sqrt{\delta} T_{n}\right)\right]=V(0,0) .
$$

Additionally, it is obvious that if $\varphi \in C_{b . L i p}(\mathbb{R})$, i.e., $|\varphi(x)-\varphi(y)| \leq C|x-y|$, then for each $t, s \in[0,1+h]$ and $x \in \mathbb{R}$,

$$
|V(t, x)-V(s, x)| \leq C \widetilde{E}[|\xi|] \sqrt{|t-s|} \leq C \sqrt{|t-s|} .
$$


In particular,

$$
|V(0,0)-V(h, 0)| \leq C \sqrt{h} .
$$

Combining (3.18), (3.23), with (3.24), we have

$$
\begin{aligned}
& \left|\hat{E}\left[\varphi\left(\sqrt{\delta} T_{n}\right)\right]-\widetilde{E}[\varphi(\xi)]\right|=\left|\hat{E}\left[V\left(1+h, \sqrt{\delta} T_{n}\right)\right]-V(h, 0)\right| \\
& \leq\left|\hat{E}\left[V\left(1+h, \sqrt{\delta} T_{n}\right)\right]-\hat{E}\left[V\left(1, \sqrt{\delta} T_{n}\right)\right]\right| \\
& +\left|\hat{E}\left[V\left(1, \sqrt{\delta} T_{n}\right)\right]-V(0,0)\right|+|V(0,0)-V(h, 0)| \\
& \leq 2 C \sqrt{h}+\left|\hat{E}\left[V\left(1, \sqrt{\delta} T_{n}\right)\right]-V(0,0)\right| .
\end{aligned}
$$

From (3.22), we obtain

$$
\limsup _{n \rightarrow \infty}\left|\hat{E}\left[\varphi\left(\sqrt{\delta} T_{n}\right)\right]-\widetilde{E}[\varphi(\xi)]\right| \leq 2 C \sqrt{h}
$$

So (3.16) is proved.

By the Lipschitz continuity of $\varphi$ and (B1), we have

$$
\left|\hat{E}\left[\varphi\left(\frac{S_{n}}{\sqrt{n}}\right)\right]-\hat{E}\left[\varphi\left(\frac{T_{n}}{\sqrt{n}}\right)\right]\right| \leq C \frac{1}{\sqrt{n}} \sum_{i=1}^{n} \hat{E}\left[\left|X_{i}-Y_{i}\right|\right] \rightarrow 0 \quad \text { as } \quad n \rightarrow \infty .
$$

Thus

$$
\lim _{n \rightarrow \infty} \hat{E}\left[\varphi\left(\frac{S_{n}}{\sqrt{n}}\right)\right]=\widetilde{E}[\varphi(\xi)], \quad \forall \varphi \in C_{b . L i p}(\mathbb{R}) .
$$

The rest of the proof is very similar to that of Theorem 3.5 in [19] and so it is omitted.

Theorem 3.4. Under the conditions of Theorem 3.3, then if $y$ is a point at which $\widetilde{v}$ is continuous, we have

$$
\lim _{n \rightarrow \infty} v\left(\frac{S_{n}}{\sqrt{n}} \leq y\right)=\widetilde{v}(y)
$$

and if $y$ is a point at which $\widetilde{\mathbb{V}}$ is continuous, we have

$$
\lim _{n \rightarrow \infty} \mathbb{V}\left(\frac{S_{n}}{\sqrt{n}} \leq y\right)=\widetilde{\mathbb{V}}(y)
$$

where $\widetilde{v}(y)=\inf _{\theta \in \Theta} E_{P}\left[I_{\left\{\int_{0}^{1} \theta_{s} d B_{s} \leq y\right\}}\right], \widetilde{\mathbb{V}}(y)=\sup _{\theta \in \Theta} E_{P}\left[I_{\left\{\int_{0}^{1} \theta_{s} d B_{s} \leq y\right\}}\right]$,

$\left\{B_{t}\right\}_{t \geq 0}$ is a 1 -dimensional Brownian motion under probability measure $P$,

$\Theta:=\left\{\left\{\theta_{t}\right\}_{t \geq 0}: \theta_{t}\right.$ is $\mathcal{F}_{t}$-adapted process such that $\left.\underline{\sigma} \leq \theta_{t} \leq \bar{\sigma}\right\}$,

$\mathcal{F}_{t}:=\sigma\left\{B_{s}: 0 \leq s \leq t\right\} \vee \mathcal{N}, \mathcal{N}$ is the collection of P-null subsets.

The proof is similar to the proof of Theorem 3.1 in [12] and so it is omitted. 


\section{ACKNOWLEDGMENTS}

This work was supported partly by the National Natural Science Foundation of China (No. 11301295), the Natural Science Foundation of Shandong Province of China (Nos. ZR2016JL002, ZR2017MA012 and ZR2015AL013), the Education Department of Shandong Province Science and Technology Plan Project (No. J16LI07), the Program for Scientific Research Innovation Team in Colleges and Universities of Shandong Province of China, and the Program for Scientific Research Innovation Team in Applied Probability and Statistics of Qufu Normal University (No. 0230518).

\section{REFERENCES}

[1] P. Artzner, F. Delbaen, J.M. Eber, and D. Heath, Coherent measures of risk, Math. Finance, 9 (1999), 203-228.

[2] Z.J. Chen, Strong laws of large numbers for capacities, arXiv:1006.0749v1, 2010.

[3] Z.J. Chen and F. Hu, A law of the iterated logarithm for sublinear expectations, J. Financ. Eng., 1 (2014), 1-23.

[4] F. Delbaen, Coherent risk measures on general probability spaces, In: Sandmann K, Schönbucher P J, Ed., Advances in Finance and Stochastics, New York: Springer-Verlag, 2002, 1-37.

[5] L. Denis, M.S. Hu and S.G. Peng, Function spaces and capacity related to a sublinear expectation: Application to G-Brownian motion paths, Potential Anal., 34 (2011), 139-161.

[6] H. Föllmer and A. Schied, Convex measures of risk and trading constraints, Finance Stoch., 6 (2002), 429-447.

[7] M. Frittelli and E. Rosazza Gianin, Putting order in risk measures, Journal of Banking \& Finance, 26 (2002), 1473-1486.

[8] M. Frittelli and E. Rosazza Gianin, Dynamic convex risk measures, In: Szegö G, Ed., New Risk Measures for the 21st Century, New York: John Wiley \& Sons, 2004, 227-248.

[9] F. Hu, Moment bounds for IID sequences under sublinear expectations, Sci. China Math., 54 (2011), 2155-2160.

[10] F. Hu and Z.J. Chen, General laws of large numbers under sublinear expectations, Communications in Statistics - Theory and Methods, 45 (2016), 4215-4229.

[11] F. Hu, Z.J. Chen and P.Y. Wu, A general strong law of large numbers for nonadditive probabilities and its applications, Statistics, 50 (2016), 733-749. 
[12] F. Hu and D.F. Zhang, Central limit theorem for capacities, C. R. Math. Acad. Sci. Paris, 348 (2010), 1111-1114.

[13] M. Li and Y.F. Shi, A general central limit theorem under sublinear expectations, Sci. China Math., 53 (2010), 1989-1994.

[14] S.G. Peng, Law of large numbers and central limit theorem under nonlinear expectations, arXiv:math.PR/0702358vl, 2007.

[15] S.G. Peng, A new central limit theorem under sublinear expectations, arXiv:0803. 2656vl, 2008.

[16] S.G. Peng, Survey on normal distributions, central limit theorem, Brownian motion and the related stochastic calculus under sublinear expectations, Sci. China Ser. A, 52 (2009), 1391-1411.

[17] S.G. Peng, Nonlinear expectations and stochastic calculus under uncertainty with robust central limit theorem and G-Brownian motion, arXiv:1002.4546v1, 2010.

[18] L.X. Zhang, Rosenthal's inequalities for independent and negatively dependent random variables under sub-linear expectations with applications, Sci. China Math., 59 (2016), 751-768.

[19] L.X. Zhang, Exponential inequalities under the sub-linear expectations with applications to laws of the iterated logarithm, Sci. China Math., 59 (2016), 25032526. 